\title{
Distinct roles for the mammalian A-type cyclins during oogenesis
}

\author{
Jenny Liao Persson ${ }^{1}$, Qi Zhang ${ }^{2}$, Xiang Yuan Wang $^{2}$, Stuart E Ravnik ${ }^{3}$, Samantha Muhlrad ${ }^{2}$ and \\ Debra J Wolgemuth ${ }^{1,2,3,4,5}$
}

${ }^{1}$ Departments of Genetics and Development, ${ }^{2}$ Obstetrics and Gynecology, ${ }^{3}$ The Center for Reproductive Sciences, ${ }^{4}$ The Herbert Irving Comprehensive Cancer Center and ${ }^{5}$ The Institute of Human Nutrition, Columbia University Medical Center, New York, New York, USA

Correspondence should be addressed to D J Wolgemuth, Department of Genetics and Development, Black Building 1613, College of Physicians and Surgeons, Columbia University, 630 West 168th Street, New York, New York 10032, USA Email: djw3@columbia.edu

(J L Persson is now at the Division of Pathology, Department of Laboratory Medicine, Lund University, Malmo, Sweden) (S E Ravnik is now at the Division of Basic Science, UT Southwestern Graduate School of Biomedical Sciences, Dallas, Texas, USA)

\begin{abstract}
There are two A-type cyclins in higher vertebrates, cyclin A1 and A2. Targeted mutagenesis has shown that cyclin A2 is essential for early embryonic development while cyclin A1 is required only for male meiosis. The embryonic lethality of cyclin A2 knockout mice has obviated understanding its role in other aspects of mammalian development, including the germ line. We reported previously that cyclin A2 expression in the male germ line is consistent with a role in both mitotic and meiotic cell cycles. Using in situ hybridization and immunohistochemistry, we now observe high levels of cyclin A2 in granulosa cells and less-abundant but readily detectable expression in ovarian and ovulated oocytes. A decrease in cyclin A2 protein was observed in oocytes from embryonic stages to post-natal and adult ovaries. Interestingly, cyclin A2 protein was nuclear in oocytes from embryonic day 13.5 to 15.5 , changing to largely cytoplasmic in oocytes from embryonic day 16.5 to post-natal and adults. Readily detectable expression of the cyclin-dependent kinases Cdk1 and Cdk2, two common partners for the A-type cyclins, was observed in granulosa cells and oocytes at all stages of folliculogenesis. Cdk1 was predominantly cytoplasmic, whereas Cdk2 was both cytoplasmic and nuclear in oocytes. No cyclin A1 expression, at either the mRNA level or the protein level was detected in either embryonic or adult ovaries, consistent with the full fertility observed in female cyclin A1-deficient mice. These results suggest that in the female germ line, cyclin A2 but not cyclin A1 has distinct roles in both mitosis and meiosis.
\end{abstract}

Reproduction (2005) 130 411-422

\section{Introduction}

Mammalian oogenesis involves a highly orchestrated series of mitotic and meiotic cell cycles. In most mammals, most female germ cells undergo a number of mitotic divisions and then enter into meiosis during embryogenesis. In the mouse, oogonia enter prophase of the first meiotic division at embryonic day 13.5 (E13.5) and quickly progress through leptotene, zygotene and pachytene stages before arresting in diplotene, at which point they are surrounded by a single layer of follicle cells (McLaren \& Southee 1997). The oocyte remains arrested in the diplotene stage and pools of resting oocytes are recruited on a cyclical basis to enter a period of oocyte and follicular growth at sexual maturity. At the end of the growth period, the oocyte is meiotically competent, that is, able to undergo the first meiotic division, only to arrest again awaiting fertilization (Taieb et al. 1997). The progression of the meiotic cell cycle in oogenesis is thus developmentally controlled and the meiotic cell cycle can arrest and resume in response to different stimuli. It is therefore important to understand the role of critical cell cycle-regulatory genes in this process.

The cyclins and cyclin-dependent kinases (Cdks) play key roles in the regulation of cellular responses to growth and passage through distinct phases of the cell cycle (reviewed by Pines 1993, Sherr \& Roberts 1995, Jackman \& Pines 1997). Activation of the kinase requires the 
association of regulatory cyclin subunits, and different cyclins preferentially complex with particular Cdk partners (Hunter \& Pines 1994). Regulation of the kinase complexes at distinct cell cycle phases is accomplished in part by differential expression, binding, localization and degradation of cyclins (Yang \& Kornbluth 1999, Miller \& Cross 2001, Murray 2004).

Among the cyclins, the in vivo function of the A-type cyclins has remained somewhat enigmatic. There are two A-type cyclin proteins in frog (Howe et al. 1995), mice (Sweeney et al. 1996) and human (Yang et al. 1997). The originally described mammalian A-type cyclin, known as cyclin A2, is widely expressed and has been studied extensively both in vitro as well as in mammalian cell lines. Cyclin A2 has been shown to be required for S-phase (Girard et al. 1991) as well as at the G2/M transition (Pagano et al. 1992) and to co-localize with sites of DNA replication in mouse cell lines (Cardoso et al. 1993). Cyclin A2 can bind and activate both Cdk1 and Cdk2 in somatic cells (Elledge et al. 1992, Kobayashi et al. 1992, Rosenblatt et al. 1992), consistent with its function in both DNA replication and onset of mitosis. An absolute requirement for cyclin A2 during development and differentiation was elegantly demonstrated by gene targeting (Murphy et al. 1997). Cyclin A2-deficiency results in embryonic lethality around the peri-implantation stage. Unfortunately, this early lethality precludes studies assessing the effects of lack of cyclin A2 in the tissues and organs of the adult animal.

In contrast to the broad distribution of cyclin A2 expression, cyclin A1 is found at highest levels, if not exclusively, in the adult testis (Ravnik \& Wolgemuth 1996, Sweeney et al. 1996). Although there have been some reports of cyclin A1 expression in other tissues (Sweeney et al. 1996, Murphy et al. 1997, Yang et al. 1997, Fuchimoto et al. 2001), any such expression is of questionable functional significance given the phenotype of the knockout of the gene encoding mouse cyclin A1 (Ccna1) (Liu et al. 1998). Mutagenesis of Ccna 1 revealed an absolute requirement for progression of male, but not female germ cells through the first meiotic division (Liu et al. 1998). Further, Cdk1 forms a complex with cyclin A1 in murine testis (Sweeney et al. 1996, Liu et al. 1998) and the association of Cdk1 with cyclin A1 is essential for M-phase promoting factor (MPF) activity (cyclin B1/Cdk1) (Liu et al. 2000). Given the highly specific expression patterns of the A-type cyclins and essential function of cyclin A1 in the male germ line, we wished to determine their cellular and developmental-specificity of expression in the female germ line and gonad. Cyclin A2 protein has been shown to be expressed in total ovary (Sweeney et al. 1996) and oocytes (Winston et al. 2000) but the relative levels of mRNA and protein among ovarian cell types and at different stages of oogenesis have not been determined. There also remains some uncertainty with regard to whether cyclin A1 is expressed in female germ cells. This is of particular interest since the frog cyclin A1 is clearly expressed in oocytes and Murphy et al. (1997) proposed that maternal cyclin A1 might be involved in enabling early mouse embryos lacking cyclin A2 to undergo a few rounds of cell division. In the present study we have examined the expression of cyclin A2 at both the mRNA and protein levels during meiotic differentiation of the female germ line. Our findings suggest a role for cyclin A2 in meiosis and mitosis of female germ cells. Furthermore, we have determined the distribution of the cyclin A2-associated proteins Cdk1 and Cdk2 during folliculogenesis in the adult ovary. Finally, we confirm by immunoblot analysis of adult oocytes from normal and cyclin A1deficient adult mice and by immunohistochemical analysis of embryonic ovaries that Ccna 1 is not expressed in oocytes at stages comparable with its expression in the male germ cells. Thus, cyclin A1 is restricted to the male germ line, consistent with the functional observations resulting from the outcome of gene targeting.

\section{Materials and Methods}

\section{Animals and tissues}

Normal tissues for in situ hybridization and immunohistochemical analyses were obtained from female C57B16/JF1 mice purchased from The Jackson Laboratory (Bar Harbor, ME, USA). All animals were housed and used in strict adherance to IACUC guidelines.

Ovaries and oviductal ampullae were fixed immediately in $4 \%$ paraformaldehyde in $1 \times \mathrm{PBS}$ overnight at $4^{\circ} \mathrm{C}$. In some experiments, ampullae were collected from animals that had been superovulated by i.p. injection of $5 \mathrm{IU}$ pregnant mare serum (PMSG) (Sigma), followed $48 \mathrm{~h}$ later by injection of $5 \mathrm{IU}$ human chorionic gonadotropin (hCG) (Sigma). The tissues were dissected $15 \mathrm{~h}$ after injection with hCG and processed as above. Cyclin A1-deficient females were obtained from the $\mathrm{Ccna}^{-1-}$ strain, maintained on a mixed C57Bl/6 6 Sv129 background, and genotyped as previously described (Liu et al. 1998).

Germinal vesicle (GV)-stage oocytes were collected from ovaries of control and $C \mathrm{Cna} 1^{-1-}$ mice stimulated with PMSG according to our standard procedures (Mutter et al. 1988). The ovaries were dissected $48 \mathrm{~h}$ after PMSG injection and oocytes released by follicle puncture into M2 medium (Special Media, Phillipsburg, NJ, USA) containing $100 \mu \mathrm{g} / \mathrm{ml}$ dibutyryl cAMP (Sigma). Oocytes at metaphase I were obtained by removing the GV oocytes from the M2 + cAMP medium and culturing them for $15 \mathrm{~h}$ in M16 medium (Special Media). Metaphase II-arrested oocytes were collected from mice superovulated as above. Oviducts were dissected $15 \mathrm{~h}$ after injection with hCG and oocytes were released into M2 medium. Cumulus cells from oocytes at all stages were dispersed with hyaluronidase and zonae were removed using acid-M2 as described by Simerly \& Schatten (1993).

For the embryonic gonad preparations, pregnant female mice from the breeding strain C57Bl6-JF1 or CCna1 ${ }^{-1-}$ mice maintained on a mixed Sv129 and C57BI6 
background were killed at daily intervals between 13.5 and 18.5 days after finding a vaginal plug (considered day E0.5). Embryos were removed from the uterus and fixed with $4 \%$ paraformaldehyde. The embryonic gonads were identified as ovary or testis by their appearance and location in the embryo, under a dissecting microscope. The embryonic ovary was removed and embedded in $1 \%$ agarose and then embedded in paraffin using our previously described standard procedures (Pesce et al. 1998). A portion of the embryonic liver was used as a source of DNA to confirm the genotype of the embryos $\left(\mathrm{CCna}^{-/-}\right.$, CCna $1^{+/-}$or CCna $^{+/+}$).

\section{Probes and reagents}

Probes corresponding to the $5^{\prime}$ end of the murine cyclin A2 cDNA were generated from plasmid p4-2 0.4 (Ravnik \& Wolgemuth 1996). Sense and antisense RNA probes for in situ hybridization were transcribed from the T7 and T3 promoters of linearized plasmids using the appropriate RNA polymerase, following the manufacturer's suggested protocols (Promega). Probes used for in situ hybridization were labeled with ${ }^{35}$ S-labeled UTP (Dupont) and hydrolyzed to approximately $0.5 \mathrm{~kb}$ in size (Cox et al. 1984).

Three sources of antibodies against cyclin A2 were used to verify the expression and subcellular localization of cyclin A2 in embryonic and adult stages of ovary. One was kindly provided by Dr Mark Carrington (Cambridge University, UK) (Sweeney et al. 1996) and used at a dilution of 1:500. Two other antibodies were purchased from Upstate Biotechnology Inc. (Lake Placid, NY, USA) and Oncogene Sciences (Cambridge, MA, USA) and were used at dilutions of 1:250 or 1:500 respectively. Cdk1 and Cdk2 antibodies were purchased from Upstate Biotechnology and were used at a dilution of 1:500. Polyclonal antibody against cyclin A1 was produced in our laboratory according to procedures previously described (Liu et al. 2000) and was used at a dilution of 1:250.

\section{In situ hybridization}

Paraformaldehyde-fixed tissues were embedded in paraffin, cut into $6 \mu \mathrm{m}$ sections and analyzed by in situ hybridization using our previously described protocols (Chapman \& Wolgemuth 1992, Ravnik \& Wolgemuth 1996). After hybridization, slides were washed at a final stringency of $0.1 \times \mathrm{SSC}$ at $65^{\circ} \mathrm{C}$ for $2 \mathrm{~h}$. Autoradiography was performed for 2 weeks. Developed slides were counterstained with hematoxylin and eosin (H\&E) and viewed on a Leitz photomicroscope under brightfield and epiluminescence optics. Photomicrographs were taken using Kodak Ektachrome 64T film.
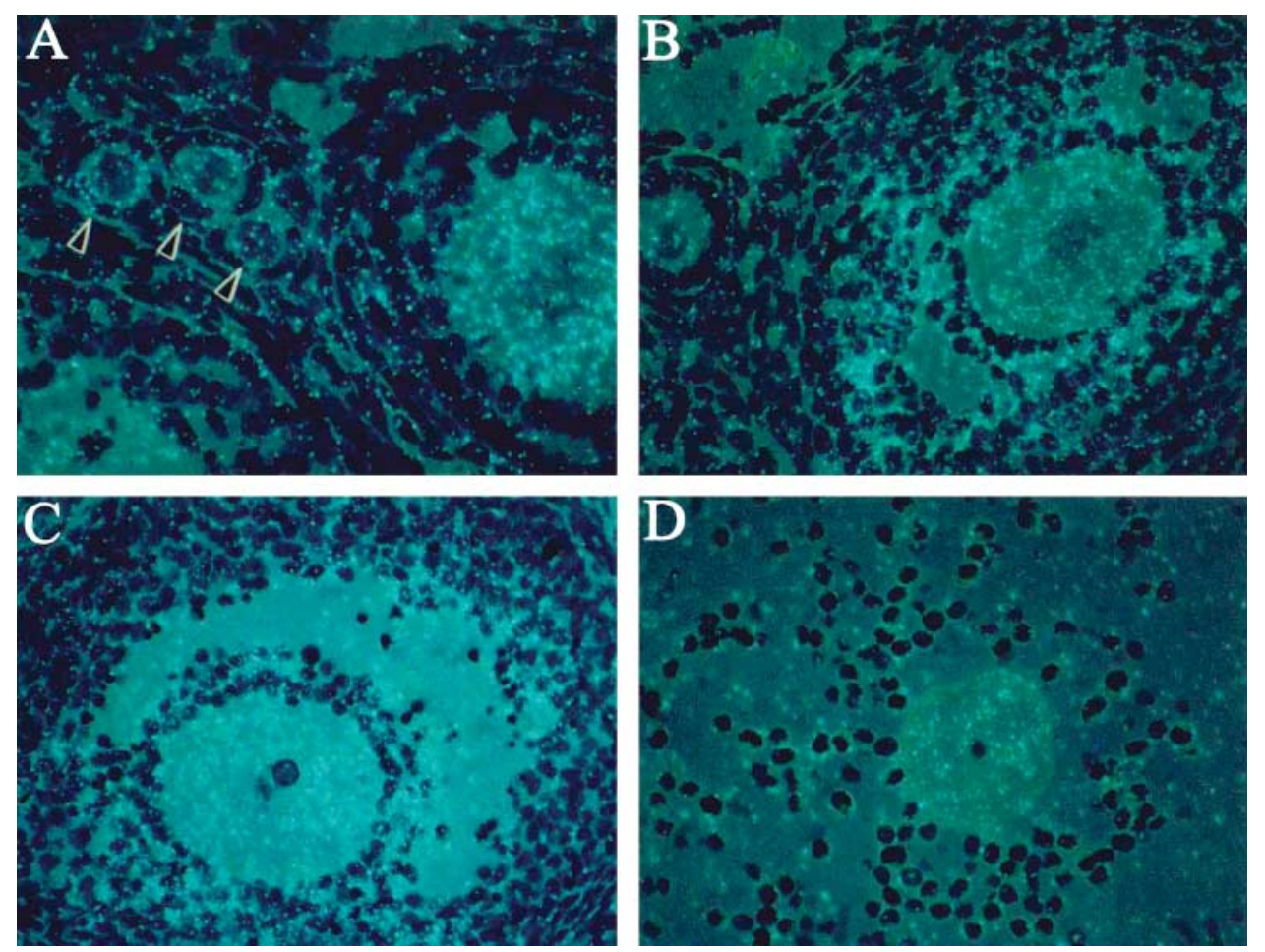

Figure 1 Localization of Ccna 1 transcripts in the adult mouse ovary by in situ hybridization. Histological sections of adult ovaries and oviductal ampullae were hybridized with ${ }^{35}$ S-labeled antisense riboprobes and photographed under epiluminescence optics. (A) Primordial follicles in stages $3 a$ to type 4 (arrowheads) and a larger, type 5a follicle on the right (see text and Pedersen \& Peters (1968) for staging). (B) Oocytes in follicular stages type 4 (left) and type 6 (right). (C) Type 7, nearly mature, Graafian follicle. (D) Cumulus-enclosed ovulated egg within the oviductal ampulla, $15 \mathrm{~h}$ after hCG. The dark spot in the center of the oocyte is a cumulus cell in a different focal plane. Exposure 3 weeks. Photographed at $\times 40$. 

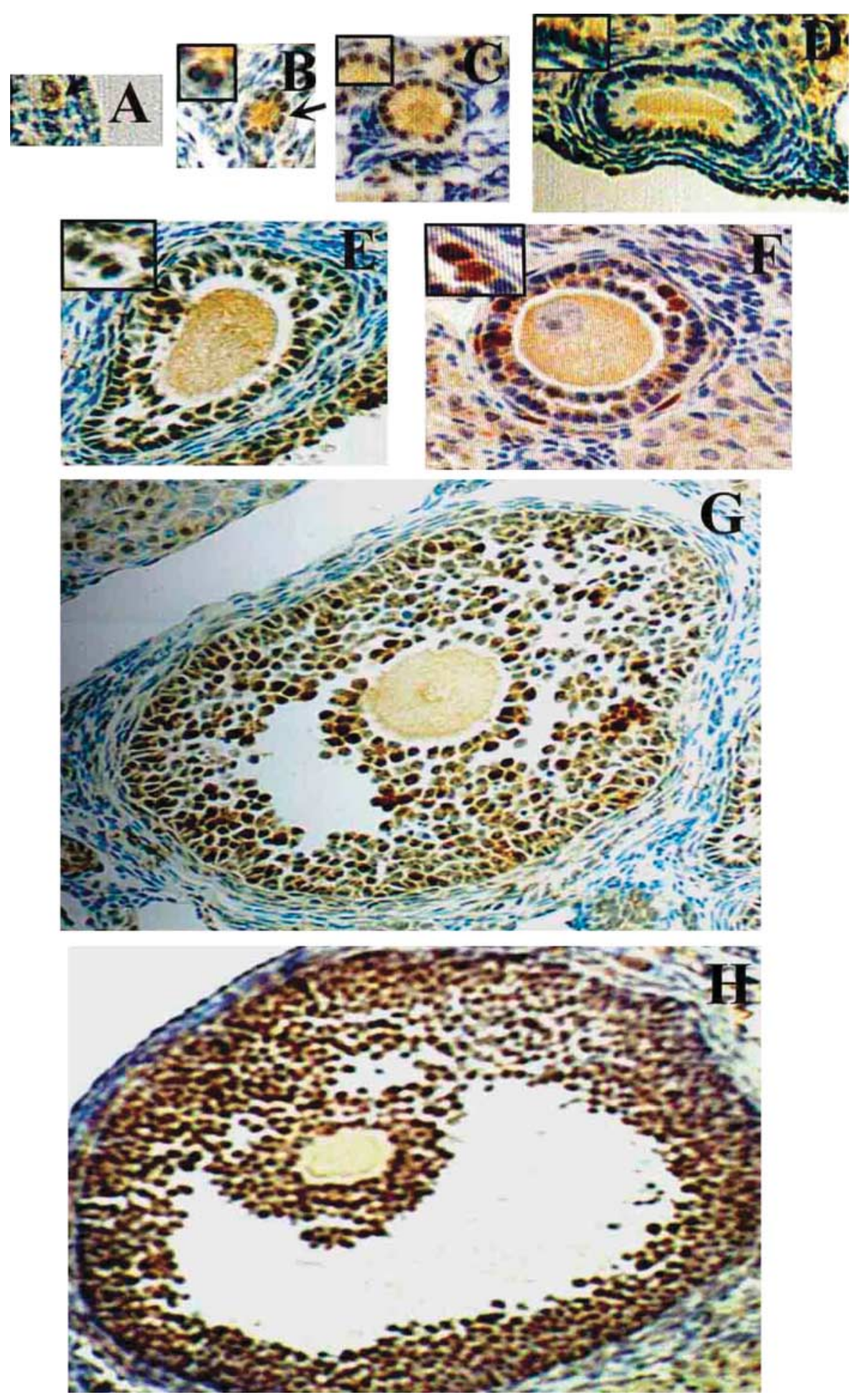

Figure 2 Expression of cyclin A2 protein in the adult mouse ovary. Histological sections of adult mouse ovary were examined for cyclin A2 expression by immunohistochemical analysis with anti-cyclin A2 antibody $(\mathrm{A}-\mathrm{H})$. Cells positive for cyclin A2 are stained with the brown $\mathrm{DAB}$ reaction product. Oocytes at different stages of growth are classified by virtue of the corresponding follicle stage. Follicles are depicted as follows: type 1 (A), type 2 (B), type $3(\mathrm{C})$, type $4(\mathrm{D})$, type $5 \mathrm{a}(\mathrm{E})$, type $5 \mathrm{~b}$ (F), type $6(\mathrm{G})$ and type $8(\mathrm{H})$. Cyclin $\mathrm{A} 2$ staining in granulosa cells in $(B-F)$ is shown in higher magnifications in insets. Photographed at $\times 60$ for follicles at stages $1-5$ and at $\times 40$ for larger follicles.

\section{Immunohistochemistry}

Paraformaldehyde-fixed, paraffin-embedded tissues were sectioned at $6 \mu \mathrm{m}$ and analyzed by immunohistochemistry using the Vectastain ABC kit (Vector Laboratories, Burlingame, CA, USA). Tissue sections were de-paraffinized in xylene, hydrated through an alcohol series, and washed with water, then boiled in antigen-retrieval buffer $(0.01 \mathrm{M}$ citrate-acetate, $\mathrm{pH}$ 6.0) in a microwave for $10 \mathrm{~min}$ and washed extensively with distilled water (Shi et al. 1991). The slides were treated with $0.03 \% \mathrm{H}_{2} \mathrm{O}_{2}$ in methanol for $20 \mathrm{~min}$ at $4{ }^{\circ} \mathrm{C}$, washed with $1 \times \mathrm{PBS}$ with $0.1 \%$ Triton $\mathrm{X}-100$ (PBSTr) and blocked for $1 \mathrm{~h}$ with $2.5 \%$ goat serum in PBSTr. Incubation with primary antibodies was carried 
out overnight at $4{ }^{\circ} \mathrm{C}$ in a humidified chamber. The slides were washed three times, $10 \mathrm{~min}$ each, with PBSTr and processed using the Vectastain $A B C$ kit, following the manufacturer's suggestions. The 3,3'-diaminobenzidine (DAB)-stained slides were counterstained with hematoxylin and viewed on a Nikon 800 photomicroscope under brightfield optics.

\section{Immunoblotting}

Fresh or frozen tissue or oocytes were lysed in lysis buffer (150 mM NaCl, $50 \mathrm{mM}$ Tris- $\mathrm{HCl}$ pH 7.5, 1\% NP-40, $10 \mathrm{mM} \mathrm{NaF}, 10 \mathrm{mM}$ phenylmethylsulfonyl fluoride and the protease inhibitors). Lysates were cleared by centrifugation at $5000 \mathrm{~g}$ for $10 \mathrm{~min}$ at $4{ }^{\circ} \mathrm{C}$. Equal amounts of proteins from each sample were then subjected to electrophoresis on a $12 \%$ SDS-PAGE gel followed by transfer to Hybond enhanced chemiluminescence (ECL) detection nitrocellulose membrane (Amersham Pharmacia Biotech). The membranes were blocked in $6 \%$ no-fat milk in Tris-buffered saline overnight and then incubated with primary antibodies (cyclin A1 at a 1:1000 dilution) for 1-2h,
Table 1 Expression of cyclin A2 protein in oocytes and ovarian follicles at pre- and post- pubertal ages.

\begin{tabular}{llcc}
\hline & \multicolumn{3}{c}{ Relative cyclin A2 expression } \\
\cline { 2 - 4 } Follicle stages present & Oocytes & $\begin{array}{c}\text { Granulosa } \\
\text { cells }\end{array}$ & $\begin{array}{c}\text { Cumulus-oocyte } \\
\text { complex }\end{array}$ \\
\hline Day 7 ovary & & & \\
Type 1-2 & ++ & ++ & \\
Type 3-4 & ++ & ++ & \\
Day 14 ovary & & & \\
Type 1-2 & ++ & ++ & \\
Type 3-4 & ++ & ++ & \\
Type 5 & ++ & +++ & \\
Type 6 & ++ & +++ & \\
Day 30 ovary & & & \\
Type 1-2 & ++ & + & \\
Type 3-4 & ++ & + & \\
Type 5 & ++ & +++ & \\
Type 6-7 & ++ & +++ & +++ \\
Day 60 ovary & & & \\
Type 1-2 & +++ & ++ & \\
Type 3-4 & +++ & ++ & \\
Type 5 & ++ & +++ & \\
Type 6-7 & + & +++ & +++ \\
Type 8 & + & +++ & +++ \\
\hline
\end{tabular}
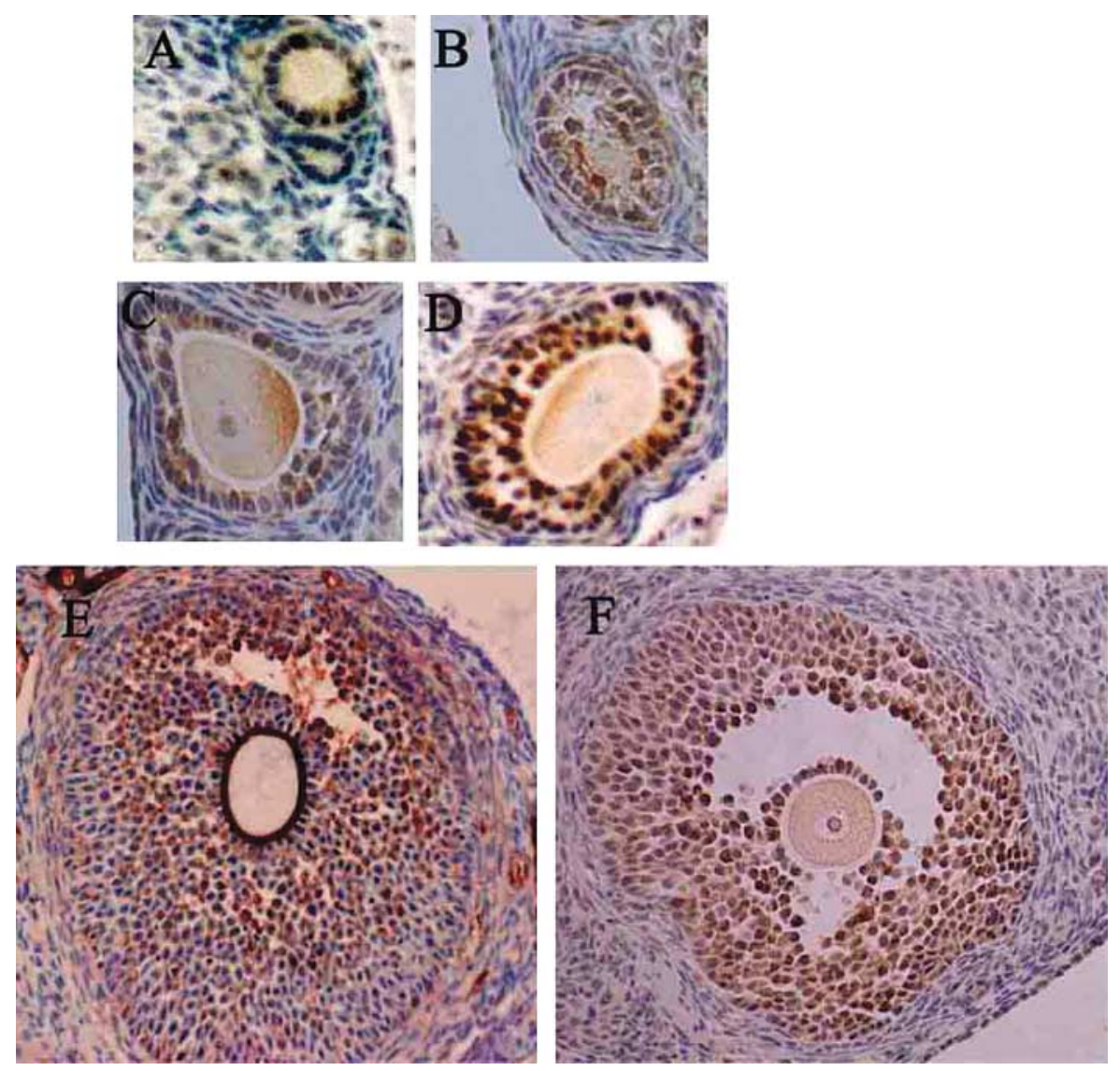

Figure 3 Expression of Cdk1 in the adult mouse ovary. The sections were processed as described in the legend to Fig. 2, with anti-Cdk1 antibody. Follicle stages are depicted as follows: type 2 and type $3(\mathrm{~A})$, type 4 (B), type $5 \mathrm{a}(\mathrm{C})$, type 5b (D), type 6 (E), and type 8 (F). Photographed at $\times 60$ for follicles at stages $1-5$ and at $\times 40$ for larger follicles. 

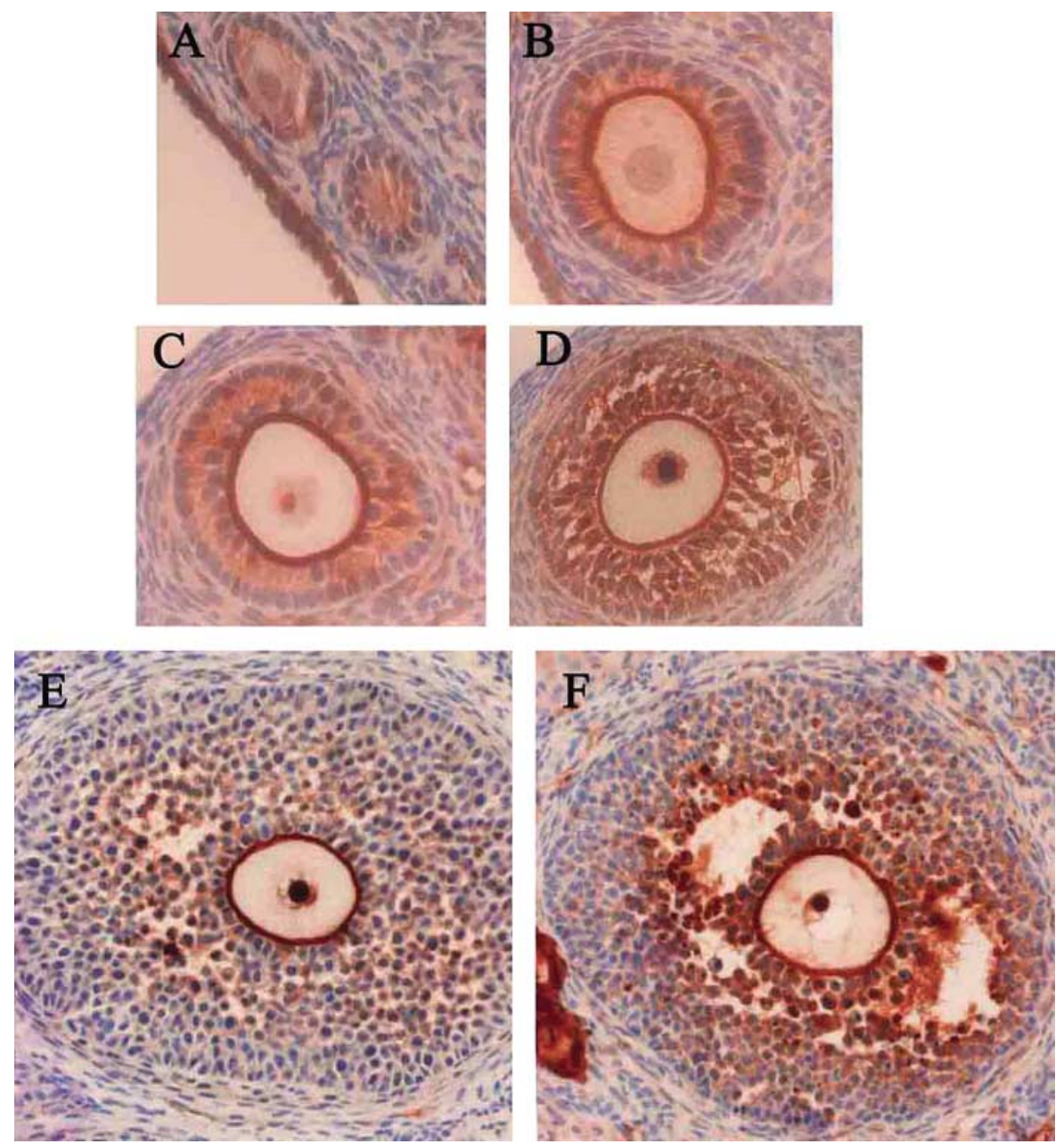

Figure 4 Expression .of Cdk2 protein in the adult mouse ovary. The sections were processed as described in the legend to Fig. 2, with anti-Cdk2 antibody. Follicle stages are depicted as follows: type $2(\mathrm{~A})$, type $3 \mathrm{~b}(\mathrm{~B})$, type $4(\mathrm{C})$, type $5 \mathrm{~b}(\mathrm{D})$, type $6(\mathrm{E})$, and type 7 (F). Photographed at $\times 60$ for follicles at stages $1-5$ and at $\times 40$ for larger follicles

followed by horseradish peroxidase-conjugated secondary antibodies (Boehringer-Mannheim, Indianapolis, IN, USA) and visualized using the ECL detection system and ECL films (Amersham Pharmacia Biotech).

\section{Results}

\section{Cyclin A2 is expressed in distinct cell types in the adult ovary}

In situ hybridization analysis and immunohistochemical methods were used to determine the cellular sites of Ccna2 expression in the adult ovary and in isolated oocytes. Oocytes in the adult ovary, which are arrested in diplotene of prophase I, can be staged according to their size and the morphological characteristics of the surrounding follicle cells (Pedersen \& Peters 1968). In situ hybridization results showed that while Ccna2 transcripts were detected in oocytes and granulosa cells at all stages of follicular development, the signals were relatively weak in the granulosa cells in early-stage follicles (stages 1-5)
(Fig. 1A). Much higher levels of signal were observed in the granulosa cells of growing follicles, particularly in the cumulus layer (cells immediately surrounding the oocyte) in stage 6-8 follicles (Fig. 1B and C). The levels of Ccna2 hybridization signal present over the oocytes remained constant in oocytes of stage 6 and 7 follicles (Fig. 1B and C) and in ovulated eggs within the oviductal ampulla (Fig. 1D). In contrast to the readily detectable Ccna2 transcripts, we were unable to detect transcripts for Ccna1 at this level of sensitivity (data not shown).

To determine whether cyclin A2 expression is regulated at the protein level, we performed immunohistochemistry on ovary sections containing various stages of follicular development (Fig. 2). The cellular distribution of cyclin A2 protein appeared to be similar to that observed for Ccna2 transcripts. In smaller follicles (type 1 and 2), cyclin A2 was clearly detected in the oocyte and at low levels if at all in the follicle cells (Fig. 2A and B). In type 3 and 4 follicles, cyclin A2 continued to be expressed in the oocytes (Fig. 2C and D) but now also in granulosa cells. 

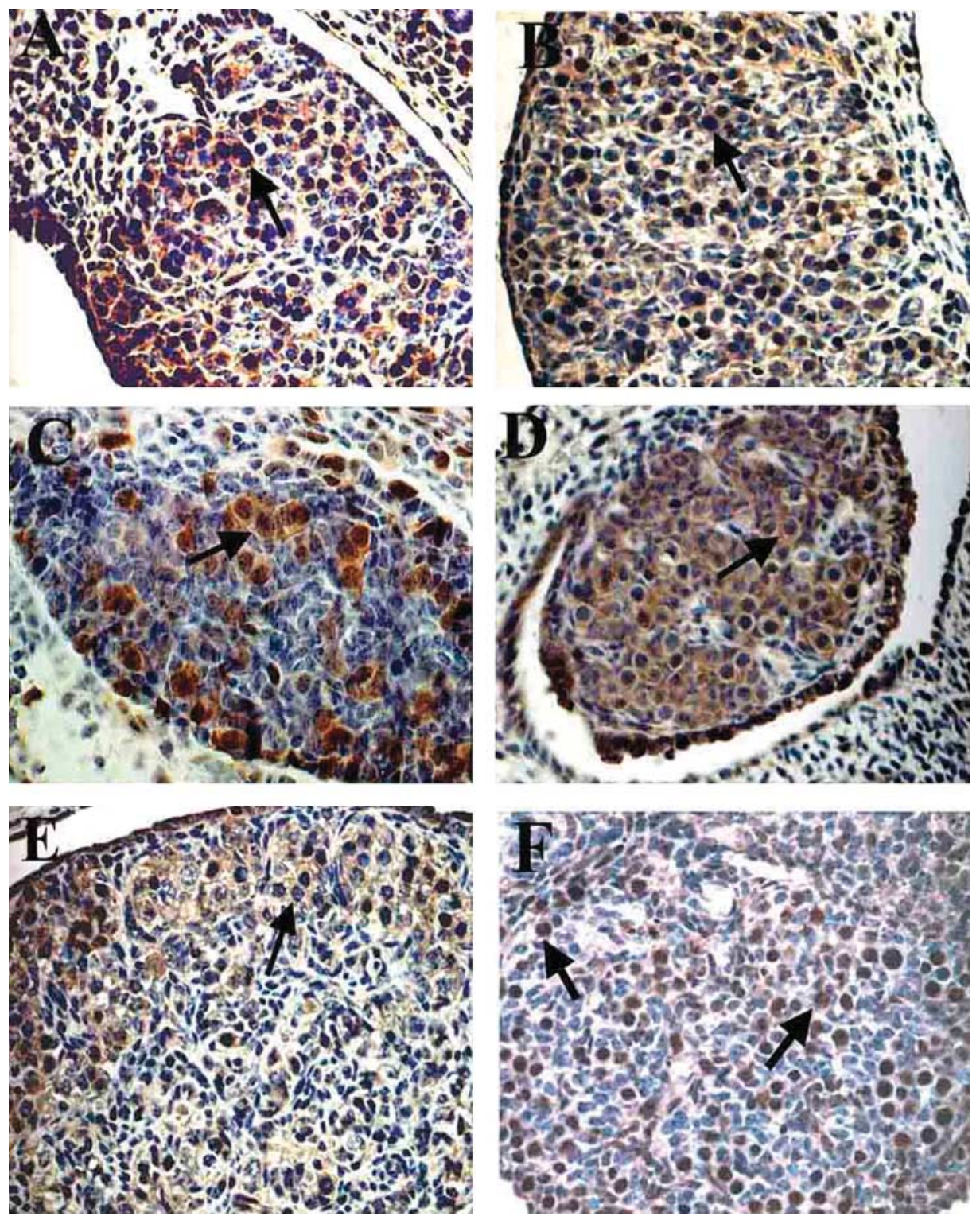

Figure 5 Localization of cyclin A2 protein in embryonic ovaries. Histological sections of embryonic ovaries were immunostained with antibody against cyclin A2 (A-E) as described above. Cyclin A2 is predominantly localized in nuclei of the germ cells (arrows) and some somatic cells of embryonic ovaries at day 13.5 (A), day 14.5 (B) and day 15.5 (C). Embryonic ovaries at day 16.5 and day 18.5 are shown in (D and E). The pachytene to diplotene oocytes no longer exhibited a predominantly nuclear staining (arrows). Embryonic ovary at day 16.5 stained with antiPCNA antibody is shown in (F). Mitotically active cells are indicated by arrows. Photographed at $\times 100$.

As follicular growth proceeded, more granulosa cells expressed cyclin A2 protein and at higher levels, while the staining intensity in the oocytes remained approximately the same in the now type $5 \mathrm{a}$ (Fig. 2E) and type $5 \mathrm{~b}$ (Fig. 2F) follicles. It is of interest to note that in large (type 6) follicles (Fig. 2G) and mature type 8 follicles (Fig. 2H), cyclin A2 protein was intensely stained in the granulosa cells and was particularly obvious in the cumulus. The levels of cyclin A2 expression in the oocytes of stage 8 follicles appeared to be much lower than those at earlier follicle stages. We also noted that some thecal cells surrounding the follicles were positive for cyclin A2 staining whereas no staining was detected in stromal cells (Fig. 2A-H). No staining was observed when control, preimmune serum was used (data not shown).

We extended this analysis to include ovaries from mice that were pre-pubertal (post-natal days 7 and 14) along with post-pubertal young adult (30 and 60 days) to determine the stage of growth and differentiation of the follicle was the primary regulator of cyclin A2 expression or if the hormonal status of animals at pre- or post-pubertal ages might also play a role. The relative levels of cyclin A2 protein in oocytes, granulosa cells and oocyte-cumulus complexes in ovaries collected at the time periods noted 
A

\title{
T Eb ov egg 1C 2C
}
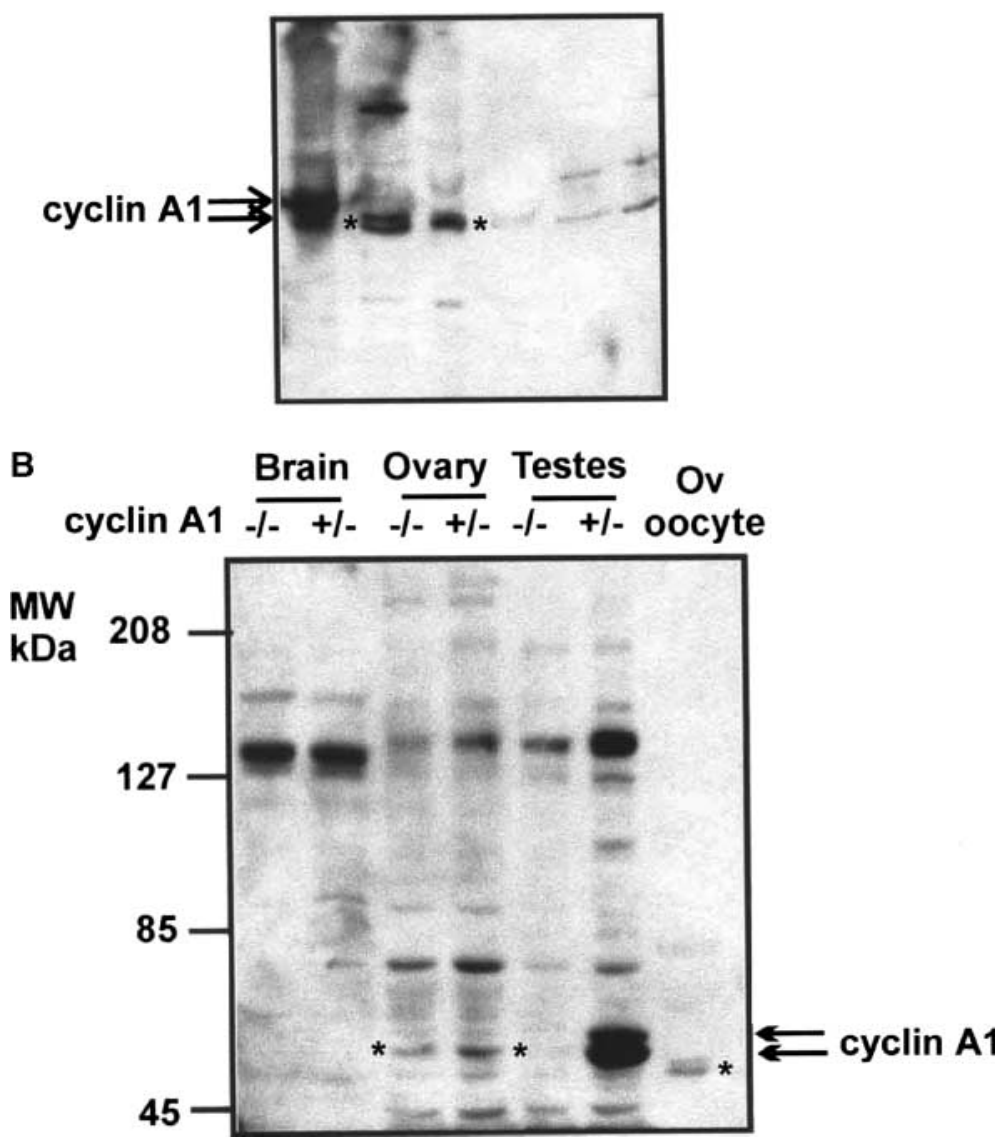

\begin{abstract}
Figure 6 Expression of cyclin A1 in $\mathrm{Ccna}^{-/-}$and Ccna $1^{+/-}$mouse tissues and oocytes. (A) Protein samples $(80 \mu \mathrm{g} / \mathrm{lane})$ were isolated from control $\left(\right.$ Ccna $\left.^{+/+}\right)$mouse testis $(\mathrm{T})$, day 12.5 embryos (Eb), young adult ovary (ov), and oocytes collected before ovulation (egg), one-cell stage (1C) and two-cell stage (2C) embryos after ovulation respectively (150 oocytes or embryos/lane) were run on SDS-PAGE, transferred to nitrocellulose, and immunoblotted with anti-cyclin A1 antibody (1:1000). (B) Protein samples (80 $\mu$ g/lane) were isolated from brain, ovary and testis of cyclin A1 knockout mice $(-/-)$ and control $(+/-)$ mouse and 120 oocytes from control mice (ov oocyte) and were run on SDS-PAGE, transferred to nitrocellulose, and immunoblotted with anti-cyclin A1 antibodies (1:1000 dilution). The size of bona fide cyclin A1 bands is indicated in the margins and the similarly migrating nonspecific bands are indicated by asterisks in the sample lane.
\end{abstract}

above were evaluated and rated from + to +++ (Table 1). The relative staining intensity for cyclin A2 in oocytes remained approximately the same in all of the follicles in pre-pubertal ovaries, which contained follicles up to early stage 6 . In the post-pubertal ovaries, levels of cyclin A2 were also more abundant in the oocytes in immature follicles but as the follicles reached stages $6-8$, the staining intensity dropped noticeably. In the granulosa cells, the patterns of cyclin A2 expression showed differentiation stage-dependent increases with follicular development in both pre-pubertal and post-pubertal ovaries. Relatively high levels of cyclin A2 were detected in the cumulus. The levels of cyclin A2 expression in granulosa cells correlated positively with the stage of follicular growth and differentiation, while in the oocyte, more mature oocytes contained less cyclin A2, regardless of the hormonal state.

\section{Localization of cyclin A2's Cdk partners in distinct cell types}

We next determined if the putative Cdk partners for cyclin A2, Cdk1 and Cdk2, were expressed in the corresponding cell types in the adult ovary. Immunohistochemical analysis revealed the expression of Cdk1 protein in oocytes and some follicle cells of type 2 and type 3 follicles and its absence in stromal cells (Fig. 3A). In type 4, 5a and 5b follicles, more follicle cells showed expression of Cdk1 (Fig. 3B-D) and it was present in the oocyte-cumulus complexes and within the oocyte of type 6 and 8 follicles (Fig. 3E and F). Occasionally, there was a non-specific staining that localized to the zona pellucida of larger oocytes (Fig. 3E), but this staining was highly variable (Fig. 3E, Fig. 4B-F). Similar to what was observed for cyclin $\mathrm{A} 2$, the staining intensity was greater in follicle cells as compared with the oocytes. However, no apparent increase in levels of Cdk1 expression was detected in follicular cells of type 8 follicles compared with the earlier stages, which was in contrast to what was observed for cyclin A2. The levels of Cdk1 expression also appeared to be similar in oocytes of different follicle stages.

Similarly to cyclin A2 and Cdk1, Cdk2 was present in granulosa cells but not in stromal cells throughout folliculogenesis (Fig. 4). In contrast to Cdk1, a significant increase in levels of Cdk2 was observed in follicle cells of type 7 compared with the earlier stages (Fig. 4A-F). This expression pattern was similar to that of cyclin A2. In oocytes, Cdk2 was both cytoplasmic and nuclear, but actually appeared to concentrate in the nucleolus of the larger oocytes in larger follicles (Fig. 4E and F). 


\section{Cyclin A2 protein expression is developmentally regulated during meiosis in oocytes}

Oocytes from post-natal or adult mouse ovaries are all in the arrested diplotene or later stages of meiosis. To determine if cyclin A2 was expressed in earlier stages of meiosis and in pre-meiotic mitotic stages, we examined cyclin A2 protein expression in embryonic mouse ovaries from E13.5 to E18.5 using immunohistochemical analysis. The staining pattern revealed that cyclin A2 was present in mitotically active somatic cells and in germ cells present in genital ridges from E13.5 to E14.5 (Fig. 5A and B). The germ cells were identified as being larger in size and having large, pale-staining, irregularly shaped nuclei on sections stained with $\mathrm{H} \& \mathrm{E}$ (data not shown). During these stages, the localization of cyclin A2 protein was predominantly nuclear. The localization of cyclin A2 continued to be predominantly nuclear in the germ cells from E15.5, when a high proportion of oocytes were entering meiotic prophase and were presumably in leptotene or zygotene (Fig. 5C), while cyclin A2 was localized in both cytoplasm and nuclei in other cell types (Fig. 5A and B). At E16.5, cyclin A2 staining remained nuclear in about $50 \%$ of the oocytes, while it became predominantly cytoplasmic in the other $50 \%$ of the oocytes (Fig. 5B). At E18.5, when the majority of oocytes are in the pachytene to diplotene to dictyate stage, cyclin A2 was present in the nuclei of only a few clusters of germ cells (Fig. 5E). The majority of clearly identifiable oocytes exhibited a predominantly cytoplasmic localization and only a weak nuclear staining pattern (Fig. 5E). Embryonic mouse ovaries from E13.5 to E18.5 were also stained for proliferating cell nuclear antigen (PCNA) with anti-PCNA antibody. As shown in Fig. $5 \mathrm{~F}, \mathrm{PCNA}$ is present in mitotically active somatic cells and in germ cells as well. A significant decrease in levels of cyclin A2 protein was observed in oocytes from the early embryonic stages to pre-natal stages, suggesting that the level of expression and cellular localization of cyclin A2 protein are highly regulated during early oogenesis.

\section{The mouse germ cell-specific A-type cyclin, cyclin A1, is not expressed in mouse oocytes}

Although the presence of cyclin A1 protein in ovulated mouse oocytes was initially reported using one set of antibodies (Sweeney et al. 1996), we have consistently been unable to detect the expression of cyclin A1 by in situ hybridization, Northern blot analysis or immunohistochemistry of adult or fetal ovaries using reagents produced in our laboratory (S E Ravnik, D Liu and D J Wolgemuth, unpublished observations). We speculated that the original observations may have detected a non-specific, crossreacting protein of the similar molecular weight on immunoblot analysis. To resolve this question definitively, we isolated ovaries and oocytes from cyclin A1-deficient mice and control mice and examined them by immunoblot analysis (Fig. 6A). Indeed, a band of approximately the same relative molecular mass as the lower band of the doublet of the bona fide cyclin A1 protein that is present in the lane of control Ccna ${ }^{+/+}$testis was detected in samples from normal ovaries, isolated eggs, and one-cell and two-cell embryos (Fig. 6A). We then isolated protein from normal and cyclin A1-deficient adult tissues (brain, ovary and testis) and isolated ovarian oocytes from cyclin A1-deficient mice (Fig. 6B). Again, a similarly migrating band was detected in the cyclin A1-deficient ovarian oocytes suggesting that this band was not cyclin A1, but represented a non-specific cross-reactivity between the cyclin A1 antibody and an unknown protein in the ovary lysates.

To test the possibility that cyclin A1 was present at early stages of meiotic prophase oocytes and turned over, we examined embryonic ovaries using anti-cyclin A1 antibodies and adult testis sections as controls. No specific staining for cyclin A1 protein was found in embryonic ovaries from E13.5 through to E18.5, the latter ages of which clearly contain pachytene and diplotene oocytes (data not shown). We therefore conclude that, consistent with the results of our targeted mutagenesis experiments (Liu et al. 1998), cyclin A1 is neither expressed during oogenesis nor required for oocyte function.

\section{Discussion}

In this study, we show that cyclin A2 is expressed in both somatic and germ cells in embryonic and adult ovaries. In embryonic ovaries, cyclin A2 protein is detected in germ cells at relatively high levels compared with somatic cells. In the adult ovary, cyclin A2 transcripts and protein are expressed in oocytes and granulosa cells with the highest levels being detected in the cumulus oophorous cells that are actively dividing. Cyclin A1 transcripts and protein are undetected in either embryonic or adult ovaries. The patterns of cyclin A2 expression observed suggest that cyclin A2 may have a function during interphase of mitotically dividing cells but also point to the interesting possibility of cyclin A2 functions during female, but not male, meiosis.

Studies of germ cell development in the mouse offer unique opportunities to explore the expression and function of cell cycle regulatory genes since these cells undergo both mitotic and meiotic divisions but exhibit striking differences in the temporal progression of these events (reviewed in Handel et al. 1999, Wolgemuth et al. 1995, Kageyama et al. 2004). In the female, the development of the ovum is interrupted at multiple points along the pathway from diploid oogonia to haploid egg (Peters 1969). Following initiation of meiosis during embryonic life, the oocyte arrests at the dictyate stage of the first meiotic prophase and remains arrested into adulthood. At this point the oocyte arrests again, and remains in metaphase II, awaiting fertilization by the sperm. In Xenopus oocytes, cyclin A2 does not appear to play a role in the oocyte (Fang \& Newport 1991, Howe et al. 1995). In fact, cyclin A2, along with cyclins A1, B1 and B2 are unlikely 
to be involved in S-phase regulation in Xenopus oocytes or cleavage stage embryos since in their absence, oocytes or egg extracts are still able to replicate DNA (Fang \& Newport 1991).

There are few studies on the expression and function of the cyclins and Cdks during differentiation and development in vivo, especially in the germ line. Previous studies in our laboratory have demonstrated a striking developmental and lineage specificity of the A- and B-type cyclins. In the female, cyclin B1 and cyclin B2 are expressed in the ovary in both somatic cells and germ cells (Chapman \& Wolgemuth 1992, 1993, 1994). The A-type cyclins, on the other hand, exhibit very different expression patterns (Ravnik \& Wolgemuth 1996, 1999, Sweeney et al. 1996, Liu et al. 1998). In the present study, we show that cyclin A2 mRNA and proteins are expressed in both somatic and germ cells in the adult ovary. In contrast, cyclin A1 is neither expressed during oogenesis nor required for oocyte function. Genetic confirmation of a corresponding function for cyclin A2 during oogenesis will await the use of conditional mutagenesis in the germ line cells, since Ccna2-null mice exhibit embryonic lethality (Murphy et al. 1997).

The high levels of expression and nuclear localization of cyclin A2 protein in the embryonic ovary suggested that it is active in germ cells as they undergo proliferation and enter into meiotic prophase. Interestingly, this robust nuclear localization changed as the oocytes progressed through meiotic prophase, such that in the pachytene to diplotene oocytes in E18.5 ovaries, cyclin A2 was predominantly cytoplasmic. A predominantly nuclear localization of cyclin A2 has been observed in male germ cells during the mitotic proliferation of spermatogonia and during the pre-leptotene stage (Ravnik \& Wolgemuth 1999), at which point cyclin A2 becomes undetected in later stage male germ cells. Late in meiotic prophase in spermatocytes, the novel A-type cyclin, cyclin A1, is then expressed, just before the first meiotic division (Ravnik \& Wolgemuth 1996, 1999, Sweeney et al. 1996, Liu et al. 1998). It is tempting to speculate that it is critical for the germ cell to exclude or prevent A-type cyclins from being active during the stages in which the cells are undergoing the specialized meiotic processes of synapsis, recombination etc. The spermatocyte might accomplish this by degrading cyclin A2 and then activating cyclin A1, while the oocyte, which does not express cyclin A1, simply sequesters cyclin A2 to the cytoplasm.

Functional inactivation of a cyclin-Cdk kinase complex by subcellular compartmentalization has precedent in other systems (reviewed in Yang \& Kornbluth 1999). Cyclin B1 is known to be sequestered in the cytoplasm and then is translocated to the nucleus just prior to the onset of MPF activity (Mitra \& Schultz 1996, Porter and Donoghue 2003). The two B-type cyclins also differ with regard to their distribution when sequestered in the cytoplasm: human cyclin B1 localizes to microtubules while cyclin B2 is found primarily associated with the Golgi
(Jackman et al. 1995, Draviam et al. 2001). A differential distribution has also been observed for the A-type cyclins in specific circumstances. While cyclin A1 is normally nuclear in late prophase spermatocytes in both mouse (Liu et al. 1998) and human (Liao et al. 2004), it was found to be predominantly cytoplasmic in leukemic cells in a transgenic mouse model (Liao et al. 2001) and in leukemic cells from patients (Ekberg et al. 2004).

In the adult ovary, the highest levels of cyclin A2 mRNA and protein were observed in the cumulus oophorous cells, those cells which are most mitotically active. At the protein level, very distinct nuclear staining of cyclin A2 was observed by immunohistochemical detection in the granulosa cells, while the pattern of staining in the oocytes appeared diffuse. The expression of cyclin A2 mRNA and protein that we observed in the proliferating granulosa cells is consistent with previous results from mammalian tissue culture cells. In numerous studies, cyclin A2 has been shown to be present at highest levels during S-phase and G2, with levels declining as mitosis begins and being undetected by metaphase (Sobczak-Thepot et al. 1993, Pines \& Hunter 1994). Indeed, we were unable to detect cyclin A2 in granulosa cells showing mitotic figures (data not shown).

In summary, cyclin A2 is expressed in both the somatic and germinal lineages in the mouse ovary (the present study) as well as in the germ cells in the testis (Ravnik \& Wolgemuth 1996). The patterns of expression suggest that cyclin A2 may indeed have a function during interphase of mitotically dividing cells but also point to the interesting possibility of cyclin A2 functions during female, but not male, meiosis. Since cyclin A1 is expressed during the meiotic division in the male (Sweeney et al. 1996), the two A-type cyclins appear to play very different roles during meiosis in the male and female.

\section{Acknowledgements}

We would like to thank Dr Mark Carrington (University of Cambridge, UK) for the kind gift of the original anti-mouse cyclin A2 antibody and Chao-Ling Kuo for help in preparation and submission of the manuscript. This work was supported in part by grants NIH HD 34915 (D J W), F32 HD07605 (S E R) and NIH T32 HD 07093 (Q Z), and grants from the STINT Foundation, the Crafoordska Foundation, and the Swedish National Research Council (J L P). The authors declare that there is no conflict of interest that would prejudice the impartiality of this scientific work.

\section{References}

Cardoso MC, Leonhardt H \& Nadal-Ginard B 1993 Reversal of terminal differentiation and control of DNA replication: cyclin A and Cdk2 specifically localize at subnuclear sites of DNA replication. Cell 74 979-992.

Chapman DL \& Wolgemuth DJ 1992 Identification of a mouse Btype cyclin which exhibits developmentally regulated expression in the germ line. Molecular Reproduction and Development 33 $259-269$. 
Chapman DL \& Wolgemuth DJ 1993 Isolation of the murine cyclin B2 CDNA and characterization of the lineage and temporal specificity of expression of the B1 and B2 cyclins during oogenesis, spermatogenesis and early embryogenesis. Development 118 229-240.

Chapman DL \& Wolgemuth DJ 1994 Expression of proliferating cell nuclear antigen in the mouse germ line and surrounding somatic cells suggests both proliferation-dependent and -independent modes of function. International Journal of Developmental Biology $38491-497$.

Cox KH, DeLeon DV, Angerer LM \& Angerer RC 1984 Detection of mRNAs in sea urchin embryos by in situ hybridization using asymmetric RNA probes. Developmental Biology 101 485-502.

Draviam VM, Orrechia S, Lowe M, Pardi R \& Pines J 2001 The localization of human cyclins B1 and B2 determines CDK1 substrate specificity and neither enzyme requires MEK to disassemble the Golgi apparatus. Journal of Cell Biology 152 945-958.

Ekberg J, Landberg G, Holm C, Richter J, Wolgemuth DJ \& Persson JL 2004 Regulation of the cyclin A1 protein is associated with its differential subcellular localization in hematopoietic and leukemic cells. Oncogene 23 9082-9089.

Elledge SJ, Richman R, Hall FL, Williams RT, Lodgson N \& Harper JW 1992 CDK2 encodes a 33-kDa cyclin A-associated protein kinase and is expressed before CDC2 in the cell cycle. PNAS $\mathbf{8 9}$ $2907-2911$.

Fang F \& Newport JW 1991 Evidence that the G-S and G2-M transitions are controlled by different cdc2 proteins in higher eukaryotes. Cell 66 731-742.

Fuchimoto D, Mizukoshi A, Schultz RM, Sakai S \& Aoki F 2001 Posttranscriptional regulation of cyclin A1 and cyclin A2 during mouse oocyte meiotic maturation and preimplantation development. Biology of Reproduction 65 986-993.

Girard F, Strausfeld U, Fernandez A \& Lamb NJ 1991 Cyclin A is required for the onset of DNA replication in mammalian fibroblasts. Cell 67 1169-1179.

Handel MA, Cobb J \& Eaker S 1999 What are the spermatocyte's requirements for successful meiotic division? Journal of Experimental Zoology $285243-250$.

Howe JA, Howell M, Hunt T \& Newport JW 1995 Identification of a developmental timer regulating the stability of embryonic cyclin A and a new somatic A-type cyclin at gastrulation. Genes and Development 9 1164-1176.

Hunter T \& Pines J 1994 Cyclins and cancer. II: Cyclin D and CDK inhibitors come of age. Cell 79 573-582.

Jackman M, Firth M \& Pines J 1995 Human cyclins B1 and B2 are localized to strikingly different structures: B1 to microtubules, B2 primarily to the Golgi apparatus. EMBO Journal 14 1646-1654.

Jackman MR \& Pines JN 1997 Cyclins and the G2/M transition. Cancer Surveys 29 47-73.

Kageyama S, Nagata M \& Aoki F 2004 Isolation of nascent messenger RNA from mouse preimplantation embryos. Biology of Reproduction 71 1948-1955.

Kobayashi H, Stewart E, Poon R, Adamczewski JP, Gannon J \& Hunt T 1992 Identification of the domains in cyclin A required for binding to, and activation of, p34cdc2 and p32cdk2 protein kinase subunits. Molecular Biology of the Cell 3 1279-1294.

Liao C, Li SQ, Wang X, Muhlrad S, Bjartell A \& Wolgemuth DJ 2004 Elevated levels and distinct patterns of expression of A-type cyclins and their associated cyclin-dependent kinases in male germ cell tumors. International Journal of Cancer 108 654-664.

Liao C, Wang XY, Wei HQ, Li SQ, Merghoub T, Pandolfi PP \& Wolgemuth DJ 2001 Altered myelopoiesis and the development of acute myeloid leukemia in transgenic mice overexpressing cyclin A1. PNAS 98 6853-6858.

Liu D, Liao C \& Wolgemuth DJ 2000 A role for cyclin A1 in the activation of MPF and G2-M transition during meiosis of male germ cells in mice. Developmental Biology 224 388-400.

Liu D, Matzuk MM, Sung WK, Guo Q, Wang P \& Wolgemuth DJ 1998 Cyclin A1 is required for meiosis in the male mouse. Nature Genetics 20 377-380.
McLaren A \& Southee D 1997 Entry of mouse embryonic germ cells into meiosis. Developmental Biology 187 107-113.

Miller ME \& Cross FR 2001 Cyclin specificity: how many wheels do you need on a unicycle? Journal of Cell Science $\mathbf{1 1 4}$ $1811-1820$.

Mitra J \& Schultz RM 1996 Regulation of the acquisition of meiotic competence in the mouse: changes in the subcellular localization of cdc2, cyclin B1, cdc25C and wee1, and in the concentration of these proteins and their transcripts. Journal of Cell Science $\mathbf{1 0 9}$ $2407-2415$

Murphy M, Stinnakre MG, Senamaud-Beaufort C, Winston NJ, Sweeney C, Kubelka M, Carrington M, Brechot C \& SobczakThepot J 1997 Delayed early embryonic lethality following disruption of the murine cyclin A2 gene [published erratum appears in Nature Genetics 199923 481]. Nature Genetics 15 $83-86$.

Murray AW 2004 Recycling the cell cycle: cyclins revisited. Cell 116 $221-234$.

Mutter GL, Grills GS \& Wolgemuth DJ 1988 Evidence for the involvement of the proto-oncogene c-mos in mammalian meiotic maturation and possibly very early embryogenesis. EMBO Journal 7 683-689.

Pagano M, Pepperkok R, Verde F, Ansorge W \& Draetta G 1992 Cyclin $\mathrm{A}$ is required at two points in the human cell cycle. $E M B O$ Journal 11 961-971.

Pedersen T \& Peters H 1968 Proposal for a classification of oocytes and follicles in the mouse ovary. Journal of Reproduction and Fertility 17 555-557.

Pesce M, Wang X, Wolgemuth DJ \& Scholer H 1998 Differential expression of the Oct-4 transcription factor during mouse germ cell differentiation. Mechanisms of Development 71 89-98.

Peters H 1969 The development of the mouse ovary from birth to maturity. Acta Endocrinology (Copenh) 62 98-116.

Pines J 1993 Cyclins and cyclin-dependent kinases: take your partners. Trends in Biochemical Science 18 195-197.

Pines J \& Hunter T 1994 The differential localization of human cyclins $A$ and $B$ is due to a cytoplasmic retention signal in cyclin B. EMBO Journal $133772-3781$.

Porter LA \& Donoghue DJ 2003 Cyclin B1 and CDK1: nuclear localization and upstream regulators. Progress in Cell Cycle Research 5 335-347.

Ravnik SE \& Wolgemuth DJ 1996 The developmentally restricted pattern of expression in the male germ line of a murine cyclin $A$, cyclin A2, suggests roles in both mitotic and meiotic cell cycles. Developmental Biology 173 69-78.

Ravnik SE \& Wolgemuth DJ 1999 Regulation of meiosis during mammalian spermatogenesis: the A-type cyclins and their associated cyclin-dependent kinases are differentially expressed in the germcell lineage. Developmental Biology 207 408-418.

Rosenblatt J, Gu Y \& Morgan DO 1992 Human cyclin-dependent kinase 2 is activated during the $S$ and G2 phases of the cell cycle and associates with cyclin A. PNAS 89 2824-2828.

Sherr CJ \& Roberts JM 1995 Inhibitors of mammalian G1 cyclindependent kinases. Genes and Development 9 1149-1163.

Shi SR, Key ME \& Kalra KL 1991 Antigen retrieval in formalinfixed, paraffin-embedded tissues: an enhancement method for immunohistochemical staining based on microwave oven heating of tissue sections. Journal of Histochemistry and Cytochemistry 39 $741-748$.

Simerly C \& Schatten G 1993 Techniques for localization of specific molecules in oocytes and embryos. Methods in Enzymology 225 516-553.

Sobczak-Thepot J, Harper F, Florentin Y, Zindy F, Brechot C \& Puvion E 1993 Localization of cyclin A at the sites of cellular DNA replication. Experimental Cell Research 206 43-48.

Sweeney C, Murphy M, Kubelka M, Ravnik SE, Hawkins CF, Wolgemuth DJ \& Carringtion M 1996 A distinct cyclin A is expressed in germ cells in the mouse. Development 122 53-64. 
Taieb R, Thibier C \& Jessus C 1997 On cyclins, oocytes, and eggs. Molecular Reproduction and Development 48 397-411.

Winston N, Bourgain-Guglielmetti F, Ciemerych MA, Kubiak JZ, Senamaud-Beaufort C, Carrington M, Brechot C \& Sobczak-Thepot J 2000 Early development of mouse embryos null mutant for the cyclin A2 gene occurs in the absence of maternally derived cyclin A2 gene products. Developmental Biology 223 139-153.

Wolgemuth DJ, Rhee K, Wu S \& Ravnik SE 1995 Genetic control of mitosis, meiosis and cellular differentiation during mammalian spermatogenesis. Reproduction, Fertility and Development 7 669-683
Yang J \& Kornbluth S 1999 All aboard the cyclin train: subcellular trafficking of cyclins and their CDK partners. Trends in Cell Biology 9 207-210.

Yang R, Morosetti R \& Koeffler HP 1997 Characterization of a second human cyclin A that is highly expressed in testis and in several leukemic cell lines. Cancer Research 57 913-920.

Received 7 March 2005

First decision 5 May 2005

Revised manuscript received 9 June 2005

Accepted 4 July 2005 